\title{
Identificación, priorización y análisis costo-beneficio de buenas prácticas ganaderas que los productores de fincas estratificadas implementan para reducir los efectos de la variabilidad climática en el municipio de Olanchito, Yoro, Honduras
}

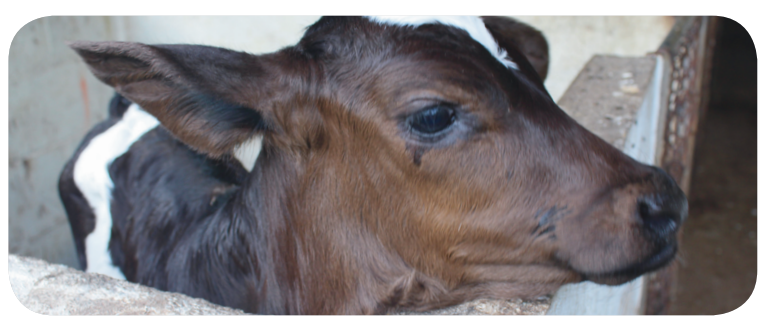

\author{
Carmen García ${ }^{1}$ \\ Claudia Sepúlveda ${ }^{2}$ \\ Manuel Gómez $z^{3}$ \\ Muhammad Ibrahim ${ }^{4}$
}

Recepción: 28/05/2018 Aceptación: 11/08/2018

\section{Resumen}

Las tendencias del cambio climático y la probabilidad creciente de nuevos cambios hacen que sea urgente adoptar prácticas y/o tecnologías que contribuyan a la resiliencia en las fincas lecheras. Mediante talleres de participación con productores $(\mathrm{n}=61)$ asociados a Centros de recolección y enfriamiento de leche, ubicados en diferentes zonas del municipio de Olanchito, departamento de Yoro en Honduras, se elaboró un catálogo de veintidos buenas prácticas, con un tiempo de implementación mayor o igual a cinco años; y llevadas a cabo por el $20 \%$ de los ganaderos.

Se priorizaron y evaluaron el 50\% de ellas con base a criterios de productividad, adaptación y mitigación. Las buenas prácticas priorizadas se clasificaron en buenas prácticas silvopastoriles, buenas prácticas de manejo y buenas prácticas de infraestructura. De este total, se seleccionaron siete para ser analizadas económicamente.

Las fincas se clasificaron en pequeñas y medianas de acuerdo con el número de cabezas de ganado y hectáreas (ha) disponibles para la ganadería, permitiendo la realización de un análisis financiero comparativo entre zonas, tipología de prácticas y tipología de fincas. Utilizando una tasa real de descuento de $4 \%$, los indicadores financieros utilizados (B/C, VAN, TIR) mostraron que las fincas pequeñas; las fincas ubicadas en la zona media y aquellas que implementaron buenas prácticas silvopastoriles fueron las más atractivas desde el punto de vista financiero. Las tecnologías priorizadas y analizadas en este artículo apuntan a mejorar la seguridad alimentaria, adaptación y mitigación de gas de efecto invernadero.

Palabras clave: Sostenibilidad, impactos climáticos, estrategias, ganancias netas.

\begin{abstract}
The trends of climate change and the increasing probability of new changes make it urgent to adopt practices and / or technologies that contribute to resilience in dairy farms. Through participatory workshops with producers $(\mathrm{n}=$ 61) associated with milk collection and cooling centers, located in different areas of the municipality of Olanchito, department of Yoro in Honduras, a catalog of twenty-two good practices was developed, with a time of implementation greater than or equal to five years; and carried out by $20 \%$ of the farmers.

$50 \%$ of them were prioritized and evaluated based on criteria of productivity, adaptation and mitigation. The good practices prioritized were classified into good silvopastoral practices, good management practices and good infrastructure practices. Of this total, seven were selected to be analyzed economically.

The farms were classified into small, and medium-size according to the number of head of cattle and hectares (ha) available for livestock, allowing the realization of a comparative financial analysis between zones, type of practices and type of farms. Using real discount rate of $4 \%$, the financial indicators used (B/C, VAN, TIR) showed that small farms; the farms located in the middle zone and those that implemented good silvopastoral practices were the most attractive from the financial point of view. The technologies prioritized and analyzed in this article aim to improve food security, adaptation and mitigation of greenhouse gas.
\end{abstract}

Key words: Sustainability, climate impacts, strategies, net gains.

1. Master Science en Economía, Desarrollo y Cambio Climático, Directora ejecutiva, Cámara hondureña de la leche (CAHLE), Tegucigalpa, Honduras; email: carmen.garcia@catie.ac.cr

2. Master Science Agroecología Tropical, Directora del Programa de Agricultura, Ganadería y Agroforestería (PRAGA), Centro Agronómico Tropical de Investigación y Enseñanza (CATIE), Turrialba, Costa Rica; email: csepul@catie.ac.cr

3. Magister Scientae en Ciencias Agrícolas, Economista-Consultor, Centro Agronómico Tropical de Investigación y Enseñanza (CATIE), Turrialba, Costa Rica; email: mgomez@catie.ac.cr

4. Ph.D. en Agricultura y Ciencias Ambientales, Director general, Centro Agronómico Tropical de Investigación y Enseñanza (CATIE), Turrialba, Costa Rica; email: mibrahim@catie.ac.cr 


\section{Introducción}

La ganadería es uno de los sistemas productivos más importantes por su contribución a la seguridad alimentaria de más de un billón de personas a nivel mundial (Acosta y Díaz 2014; FAO 2014) y constituye el motor fundamental del desarrollo económico de los países en vía de desarrollo. A lo largo del istmo centroamericano, el área de pasturas abarca un área cercana a los 13.2 millones de hectáreas (Pezo 2009), contribuye al 1.3\% del producto interno bruto regional (PIB), convirtiéndose desde el punto de vista económico en el sector agropecuario y medio de vida más importante (Acosta y Díaz 2014).

En Honduras, el sector pecuario favorece la generación de más de cuatrocientos mil empleos directos, activando la economía y contribuyendo con aproximadamente el 13\% del PIB agropecuario (Sánchez 2014). Sin embargo, el sistema ganadero predominante es de tipo extensivo o tradicional, implementado por más de 350 mil familias productoras (Aguilar et al. 2010). El manejo se caracteriza por ser deficiente, ocasionando la degradación de suelos por efectos del sobrepastoreo y malas prácticas de manejo como quemas, tratamiento inadecuado de los residuos sólidos y líquidos (SERNA
2012; Sánchez 2014), las cuales se acentúan con procesos de deforestación que son recurrentes; dando como resultado una baja rentabilidad en las fincas (Swisscontact 2014) e inestabilidad en la producción.

Los riesgos asociados al clima se encuentran intrínsecamente relacionados con el sistema de producción ganadera (Riera y Pereira 2013). El sector pecuario emite gases de efecto invernadero (metano $14,5 \%$ y óxido nitroso 7,2\%) (SERNA 2012; Sánchez 2014), pero a su vez, la variabilidad climática repercute en aspectos que tienen que ver con la disponibilidad de alimento (Craine et al. 2010, Hatfield et al. 2011, Izaurralde et al. 2011), agua, peso y salud de los animales y producción (Collier y Zimbelman 2007). Ello tiene consecuencias en la calidad de la leche (Nardone et al. 2010); y por consiguiente, un aumento en los costos de alimentación, problemas reproductivos y aumento de enfermedades transmitidas por vectores (Rust y Rust 2013).

Según el informe de brecha de adaptación publicado por el Programa de las Naciones Unidas para el Desarrollo (UNEP 2016), los países en desarrollo necesitarán entre 140 y 300 mil millones de dólares por año hasta el año 2030 para adaptarse al cambio climático. Ante este panorama se requiere fomentar el

Identificación, priorización y análisis costo-beneficio de buenas prácticas ganaderas que los productores de fincas estratificadas implementan para reducir los efectos de la variabilidad climática en el municipio de Olanchito, Yoro, Honduras 
manejo sostenible de la actividad pecuaria con la implementación de sistemas silvopastoriles y buenas prácticas ganaderas, tecnologías que han sido evidenciadas por los beneficios económicos y ambientales que proveen, apoyando en la reducción de riesgos a nivel de finca. El presente artículo presenta la percepción de los ganaderos en cuanto a la variación climática, los impactos generados por la actividad productiva, las prácticas y/o tecnologías utilizadas más importantes para prevenir y mitigar los efectos adversos; y los costos y beneficios que estas brindan permitiendo descubrir su contribución a la rentabilidad de los hatos. Asimismo, incluye información de la estimación de los beneficios económicos de la implementación de las tecnologías.

\section{Materiales y Métodos}

El estudio fue realizado en Olanchito, el segundo municipio más extenso $(2028 \mathrm{~km} 2)$ (INE 2013) del departamento de Yoro, Honduras. Está ubicado en la región agroindustrial del norte del país, entre las coordenadas $15^{\circ} 29^{\prime} 00^{\prime \prime} \mathrm{N}$ y $86^{\circ} 35^{\prime} 00^{\prime \prime}$. Al norte está rodeado por la cordillera de Nombre de Dios y al sur por la Sierra de la Esperanza, prolongación de la de Sulaco; y en el centro, por la parte alta y media del Valle del Aguan (PEDM 2012).
Como actividad económica, la producción ganadera ocupa el primer lugar entre las actividades locales (ICF-TNC 2009), con un promedio de 40 millones de litros de leche, producidos anualmente, y que son vendidos a empresas como Leche y Derivados de Honduras (LEYDE), Lácteos de Honduras S.A. (LACTHOSA) y procesadoras artesanales.

El desarrollo de la actividad pecuaria permitió el establecimiento de centros de recolección y enfriamiento de leche (CREL) a partir del 2004, cuya función principal es mantener en condiciones de temperatura e higiene aceptables la leche cruda para, posteriormente, ser entregada a la industria. Además, facilitan la asociatividad y organización de los productores (en su mayoría pequeños y medianos), los cuales se asocian a cada centro de acopio dependiendo de la distancia con las fincas ya que, mejores condiciones de accesibilidad les permite un menor tiempo entre el ordeño y la entrega de la leche (Barrios 2008).

En términos generales, el desarrollo de la ganadería en el municipio ha pasado de producir artesanalmente a insertarse en la cadena agroindustrial láctea pasteurizadora a nivel nacional, que, en comparación con el sector artesanal, recibe ingresos más altos. Sin embargo, la oferta de leche es marcadamente estacional, lo que quiere decir que experimenta 
drásticas reducciones en la época seca; pero también cuando los inviernos son copiosos y vienen acompañados de periodos de nubosidad largos, debido a que la oferta forrajera y los ciclos reproductivos son afectados por sequías o excesos de humedad (Barrios 2008).

\section{Tamaño y selección de la muestra}

Se obtuvo una lista de 18 centros de recolección y enfriamiento de leche activos y distribuidos en diferentes zonas (alta, media y montañosa) con un promedio de 20 productores por centros de recolección y enfriamiento de leche. Los datos de producción y calidad fueron brindados por la industria Leche y Derivados de Honduras (LEYDE). Una vez obtenida la información se identificaron y seleccionaron los centros con mayor producción de acuerdo con la zona (4): uno en la montaña, uno en la zona media del valle y dos en la zona alta, ya que es donde se concentra la mayoría de los productores para poder implementar talleres de trabajo.

De la lista total de productores de los centros seleccionados con mayor producción, se seleccionó una muestra al azar del 50\%, distribuida proporcionalmente al número de socios de cada centro para participar en los talleres. La muestra seleccionada es considerada suficientemente grande de acuerdo con el teorema central del límite que establece treinta o más (Di Rienzo et al. 2011). En cada taller se hizo una presentación donde se profundizó en el trabajo de investigación a realizar; se hizo una nivelación de conceptos y se aplicó una entrevista semiestructurada con el objetivo de identificar y priorizar las buenas prácticas que los productores implementan en sus fincas para hacerle frente a la variación climática. De la lista total de buenas prácticas, once se consideraron de mayor importancia; y de éstas se seleccionó el 64\% para ser analizadas económicamente. Esta selección se basó en criterios como tiempo y recursos económicos para llevar a cabo el proceso de análisis.

Para la obtención de la información se utilizó una metodología basada en el marco de priorización de inversiones en agricultura sostenible adaptadas al clima (MPASAC), utilizada por el Centro Internacional de Agricultura Tropical (CIAT) ubicado en Colombia (CCAFS 2015). De acuerdo con la metodología, primero se elaboró una lista larga de buenas prácticas ganaderas y silvopastoriles que estaban siendo utilizadas por los productores en la zona con el apoyo de una encuesta estructurada.

Luego se realizaron talleres de participación en las instalaciones de cada centro de acopio seleccionado. Se identificaron y priorizaron las prácticas utilizadas por al menos un $20 \%$ de los productores durante un periodo mayor o igual a cinco años, que es el lapso requerido para recuperar la inversión que se realiza 
en las fincas (Villanueva et al. 2010). Estas se evaluaron según su aporte a la productividad de las fincas, adaptación a las variaciones del clima y mitigación de los efectos adversos provocados por esta. Posteriormente, se elaboró una línea de tiempo en conjunto con los ganaderos para conocer los principales eventos climáticos y los impactos generados por estos en la producción ganadera durante la década 2007-2016.

Finalmente, se realizó un análisis económico para evaluar la rentabilidad de las fincas según las prácticas priorizadas. Para esto se diseñó y aplicó una encuesta semiestructurada donde se levantó información de costos (fijos y variables) e ingresos derivados de la actividad ganadera. Los ingresos por finca fueron calculados tomando en cuenta dos factores: la producción de leche anual y venta de animales.

Los costos de producción involucran los costos variables y fijos que se generan en cada finca. Los costos fijos son los que no varían independientemente de los niveles de producción porque se derivan de inversiones que tienen una vida útil de varios años, tal es el caso de maquinarias y equipos e inversiones fijas. Los costos variables varían de acuerdo con los niveles de producción (Horngren et al. 2007). Por ejemplo, los gastos en manejo zoosanitario, medicamentos, mano de obra eventual y el mantenimiento de cada una de las prácticas silvopastoriles (fertilizantes, herbicidas, etc.).

\section{Análisis ex ante}

El tipo de análisis utilizado en esta investigación (ex ante) se basa en las proyecciones futuras de costos e ingresos. Las evaluaciones económicas son una forma de comprender mejor el impacto que puede generar una práctica, así como su adopción bajo condiciones cambiantes; por ejemplo, el efecto del cambio climático (Daigneault et al. 2016). Este análisis sirve para que los tomadores de decisión evalúen las medidas de inversión (Birol et al. 2010 ) y para que los productores mejoren la resiliencia de su actividad (Dittrich et al. 2016).

Para la construcción del análisis se elaboró un desarrollo biométrico de los hatos en la que se cuantifica las compras, ventas, muertes de animales, entradas y salidas por concepto de venta de carne y leche, gastos en mantenimiento, alimentación, medicinas, etc.

Finalmente, se evaluó la rentabilidad financiera mediante flujos de caja los cuales son un resumen de las entradas y salidas de efectivo durante la ejecución de la actividad ganadera (Horngren et al. 2007). Se basaron en tres análisis comparativos: tipología de finca, zona climática y tipología de prácticas buenas prácticas silvopastoriles (SSP), buenas prácticas de manejo (BPM) y buenas prácticas de infraestructura $(\mathrm{BPI})^{5}$, utilizando los parámetros costos totales de producción e ingresos percibidos por la actividad pecuaria.

5. Dentro del documento, los autores también se referirán a estos términos mediante sus acrónimos. 
La viabilidad de los análisis fue determinada por indicadores financieros como el valor actual neto (VAN), la relación beneficio/costo (B/C) y la tasa interna de retorno (TIR), utilizados por diferentes autores para determinar la rentabilidad en tecnologías alternativas de producción y conservación (Balana et al. 2012; Bizoza y De Graaff, 2012; Prabuddh y Suresh, 2014).

La tasa de cambio utilizada fue de L23.48 frente a $\$ 1.00$ de acuerdo con el Banco Central de Honduras (BCH 2017). Se trabajó con una tasa de descuento real del $4 \%$, ya que las proyecciones y flujos de caja se trabajaron utilizando precios actuales netos de inflación; para su cálculo se utilizó la fórmula sugerida por Rose et al. (1989): [(1+tasa nominal) / (1+tasa de inflación)-1]. En la tabla 1 se indican las fórmulas utilizadas para el análisis financiero (Ver tabla 1).

\section{Resultados y Discusión}

Los ganaderos tienen conocimiento de la variabilidad en el clima, los problemas que acarrea en la actividad y sus consecuencias. El $61 \%$ menciona que han experimentado un retraso en la llegada del invierno, lluvias intensas y un aumento en las temperaturas acompañada de veranos largos (Figura 1). Los resultados son concordantes con los encontrados en otras investigaciones sobre cambio climático realizados en América Central. En este sentido Sepúlveda et al. (2008) encontraron que un número significativo de productores en Nicaragua (24\%) ( $n=60)$ y Costa Rica $(34 \%)(n=50)$ han observado cambios en la temperatura y alteraciones con respecto a la duración de la estación seca y lluviosa.

Tabla 1. Fórmulas de cálculo de los indicadores económicos para valorar la rentabilidad de la actividad ganadera de las fincas consideradas

\begin{tabular}{|c|c|c|c|}
\hline INDICADORES ${ }^{*}$ & $\begin{array}{l}\text { Valor actual neto } \\
\text { (VAN) }\end{array}$ & $\begin{array}{c}\text { Relación beneficio/ } \\
\text { costo }(B / C)\end{array}$ & $\begin{array}{l}\text { Tasa interna de } \\
\text { retorno (TIR) }\end{array}$ \\
\hline FÓRMULAS $^{\star *}$ & $\mathrm{VAN}=\frac{\sum(\mathrm{Bn}-\mathrm{Cn})}{(1+\mathrm{i})^{\mathrm{n}}}$ & $\mathrm{B} / \mathrm{C}=\frac{\sum \mathrm{Bn} /(1+\mathrm{i})^{\mathrm{n}}}{\sum \mathrm{Cn} /(1+\mathrm{i})^{\mathrm{n}}}$ & $\mathrm{TIR}=\sum \frac{\mathrm{Bn}-\mathrm{Cn}}{(1+\mathrm{i})^{\mathrm{n}}}=0$ \\
\hline
\end{tabular}

\section{Nota:}

${ }^{\star}$ B/C: índice para evaluar la rentabilidad de las inversiones; VAN: flujo incremental de beneficios netos generados por las alternativas que se comparan a lo largo de su ciclo de vida; TIR: tasa de descuento que hace que el VAN sea igual a 0 .

\footnotetext{
${ }^{*} \mathrm{Bn} /(1+\mathrm{i})^{\mathrm{n}}=$ Beneficios actualizados; $\mathrm{Cn} /(1+\mathrm{i})^{\mathrm{n}}=$ Costos actualizados; $(1+\mathrm{i})^{\mathrm{n}}=$ Factor de actualización; $\mathrm{i}=$ Tasa de descuento anual; $\mathrm{n}=$ Años de proyección del flujo de caja
} 


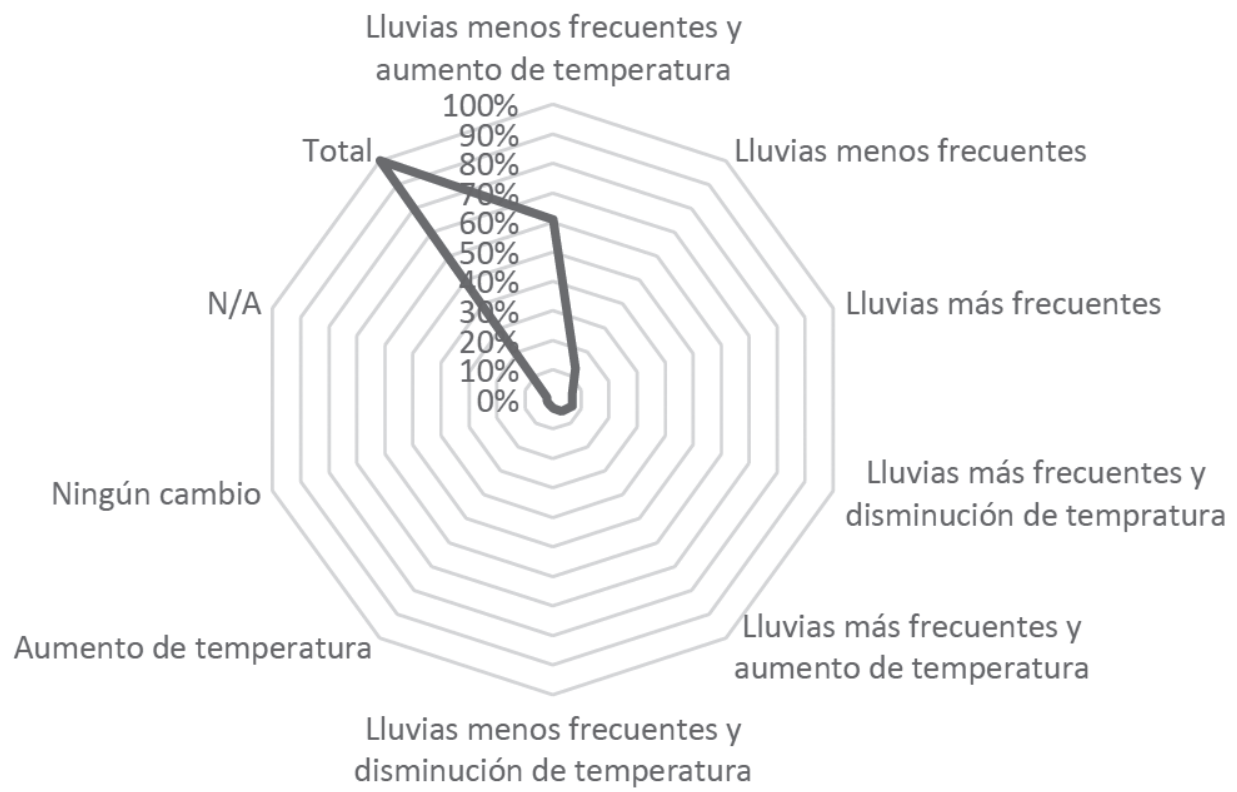

Figura 1. Percepción de los productores con respecto a los cambios en el clima en el municipio de Olanchito, Yoro, Honduras.

Independientemente de los cambios experimentados, la mayoría de los productores confirma que existe una variación en el clima, que relacionan con un incremento o disminución de calor o lluvias. Sin embargo, existen variables que pueden influir en cómo los productores perciben esos cambios. Por ejemplo, la ubicación de las fincas con respecto a las ciudades, la educación de los productores, los niveles de ingresos (Andrade 2012), la edad y la experiencia (Hartter et al. 2012), entre otros.

\section{Impactos en la producción láctea percibi- dos por cambios en el clima}

En la zona montañosa los productores indicaron que deslizamientos provocados por lluvias extremas han obstaculizado los caminos, im- pidiendo el paso a las cisternas que recolectan la leche; y con ello, han provocado la pérdida de ingresos pues la leche cruda no llega a su destino final. Asimismo, se han experimentado olas de frío y lluvias intensas acompañadas de inundaciones; veranos prolongados con aumentos de temperatura severos, variaciones que estimulan la incidencia de plagas y enfermedades, reduciendo el pastoreo y el peso animal y, en algunos casos, causan la muerte de estos últimos. Efectos idénticos se han observado en otras zonas de Centroamérica. Por ejemplo, productores ganaderos de Nicaragua y Costa Rica señalaron un incremento en el daño de las pasturas causado por diversas plagas (Sepúlveda et al. 2008). 
En la zona media, el problema de mayor afectación es la duración de la época lluviosa. Los productores manifestaron que los veranos son muy cortos, derivando bajas en la producción, deterioro en la calidad de los pastos, pérdida de animales y, por ende, de las ganancias. Los animales hacen frente a los periodos desfavorables a través de modificaciones fisiológicas y de comportamiento. Así, en la mayoría de los casos al encontrarse fuera de la denominada zona termoneutral, su respuesta se manifiesta en cambios en los requerimientos nutricionales, provocando una reducción en su desempeño productivo (Escobar et al. 2008).

En la parte alta del valle, donde predomina un clima muy seco, se da una desventaja para los productores pues el principal problema radica en la escasez de agua, provocada por las sequías o por la prolongación de la época seca con alzas extremas de temperaturas. Este es un factor crítico, ya que el agua es indispensable para el desarrollo de las pasturas $y$, por consiguiente, la sostenibilidad de los niveles de producción. Los principales impactos, según la opinión de los productores, incluyen la disminución del rendimiento de pastos, la propagación de plagas, pérdida de peso y retraso de ciclos productivos en las vacas. Los efectos son similares a los obtenidos en otros estudios que demuestran el efecto que tiene la variabilidad climática sobre la ganadería, que incide en factores como la reproducción, el crecimiento y rendimiento del animal, la cantidad y calidad de alimentos como pastos, forrajes, granos y la incidencia de plagas, parásitos y enfermedades en el ganado (Seo et al. 2010).

\section{Prácticas silvopastoriles (SSP)}

Según los resultados de las encuestas de la muestra analizada, las principales prácticas implementadas en sistemas silvopastoriles (SSP) (Figura 2), corresponden a los árboles dispersos en potreros que están presentes en la mayoría (62\%) de las fincas; las cercas vivas en el 46\% (la especie predominante es Gliricidia sepium,), las pasturas mejoradas en el 39\% y la reforestación de árboles en el 39\%. Según algunos estudios (Villanueva et al. 2010), la implementación de estas prácticas de manera adecuada y conjunta contribuyen a una mayor producción de leche sostenible y resiliente a cambios en el clima. Ecológicamente hablando, una buena combinación de cobertura arbórea con pasturas reduce la escorrentía superficial (Ríos et al. 2007), incrementa la biodiversidad (Sáenz et al. 2007) y logra un mayor secuestro de carbono (Ibrahim et al. 2007) que en pasturas degradadas o con ausencia de árboles (Ver figura 2).

Del conjunto de prácticas identificadas dentro de los SSP, un 36\% de los productores consideró la implementación de pastos de corte y un $28 \%$ las pasturas mejoradas como dos de las medidas de mayor importancia para sus 


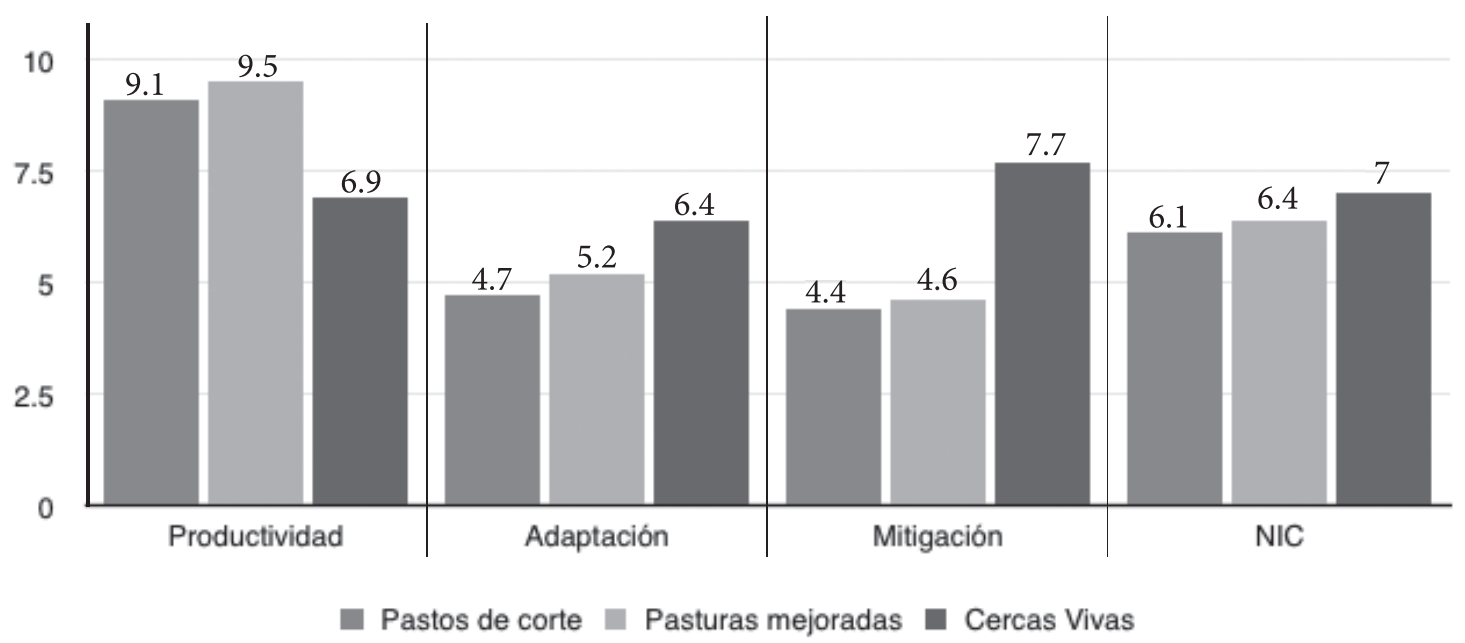

Figura 2. Prácticas identificadas e implementadas en sistemas silvopastoriles según la antigüedad de uso en el municipio de Olanchito, Yoro, Honduras.

AD: árboles dispersos; CV: cercas vivas; PM: pasturas mejoradas; RF: reforestación; AF: árboles frutales; PC: pastos de corte; BP: bancos de proteína y CRV: cortinas rompe vientos.

fincas, ya que, de acuerdo con sus criterios, estas contribuyen, entre otros beneficios, a mejorar las condiciones y la alimentación del ganado durante todo el año; aumento de la capacidad de carga, incremento en la producción de leche y aumento de los ingresos.

Según Villanueva et al. (2010), a los beneficios anteriores hay que sumar el hecho de que se evita la degradación en periodos críticos (sequías y lluvias prolongadas), reduciendo la presión en los pastos. Las cercas vivas establecidas en el $8 \%$ de las fincas conllevan, según los productores, beneficios que radican en brindar sombra al ganado, contribuyendo al confort animal y disminuyendo el estrés térmico, función apreciada por productores ga- naderos en otros estudios (Mosquera 2010). Además, los productores consideran que su implementación les permite reducir costos futuros, puesto que no se tienen que estar reemplazando postes todos los años. En este sentido, Villanueva et al. (2008a) manifiestan que el establecimiento de cercas vivas representa un ahorro del 16\% en comparación con el uso de cercas muertas.

\section{Buenas prácticas de manejo (BPM)}

Según los resultados de las entrevistas, un 50\% de las BPM mencionadas por los productores y con un uso mayor o igual a cinco años son las más utilizadas (Figura 3). En orden descendente, el ordeño limpio es la práctica más 
implementada (72\%), seguida de la división y rotación de potreros (69\%), la cual según Banegas et al. (2013) es una de las BP aplicada con mayor frecuencia en las fincas ganaderas de Honduras. De acuerdo con Villanueva et al. (2008b), esta es una práctica de fácil adopción en algunos lugares del país como Copán por el impacto visible en la productividad de las fincas.

Las prácticas que cuentan con niveles bajos de implementación son el buen manejo del estiércol, los bloques nutricionales, el ensilaje, la henificación y prácticas que promue- ven la protección del suelo. Podría pensarse que una de las principales razones de la baja implementación de estas prácticas se debe a la falta de asistencia técnica y conocimiento que tienen los productores (preparación, costos y beneficios).

En el caso de la henificación, la limitación más importante puede ser los altos costos de inversión que requiere para poder ser implementada, mayor requerimiento de mano de obra, acceso al crédito, entre otras (Calle et al. 2009) (Ver figura 3).

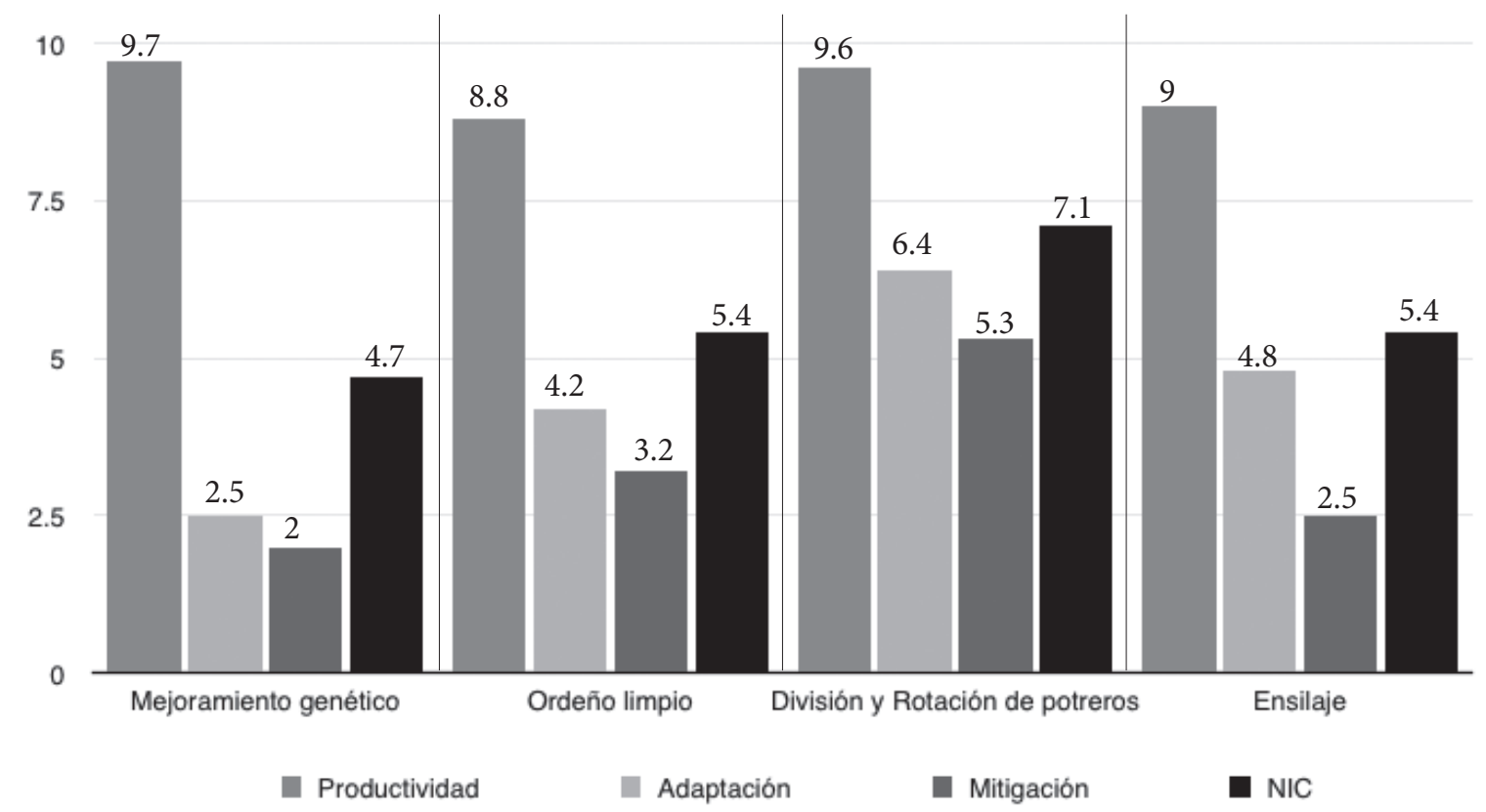

Figura 3. Buenas prácticas de manejo identificadas e implementadas de acuerdo con la antigüedad de uso en el municipio de Olanchito, Yoro, Honduras.

OL: ordeño limpio; DRP: división y rotación de potreros; NQ: no quemas; MG: mejoramiento genético; CFA: cercado de las fuentes de agua; ME: manejo del estiércol; BN: bloques nutricionales; EN: ensilaje; HE: henificación y PES: prevención erosión del suelo. 
Algunas de las prácticas descritas (pasturas mejoradas, árboles dispersos, pastos de corte, cercas vivas, ensilaje) en los dos apartados anteriores (SSP y BPM), fueron identificadas y priorizadas también por su aporte para enfrentar las variaciones del clima en otras fincas de Honduras y Nicaragua, mediante procesos participativos (Hernández et al. 2014). Esto demuestra que la experiencia de los productores contribuye al conocimiento y a reducir la vulnerabilidad de los sistemas productivos ante el cambio climático.

\section{Buenas prácticas en infraestructura (BPI)}

El tercer grupo de prácticas analizado corresponde a las BPI. Los resultados obtenidos indican que las salas de ordeño son el componente principal, ya que el $74 \%$ de los pro- ductores indicó que las tenía desde hace más de cinco años. Un 18\% de ellos las considera como las más importantes, esto porque es uno de los requerimientos de higiene que la industria exige para poder comprar la leche (Ver figura 4).

\section{Evaluación de prácticas priorizadas me- diante los pilares de productividad, adapta- ción y mitigación}

En la figura 5 se muestran los resultados de la evaluación cualitativa que los productores realizaron a cada una de las prácticas priorizadas. Los resultados indican un mayor aporte a las cercas vivas, la división y la rotación de potreros, un espacio adecuado para el equipo de trabajo del personal y las cosechas de agua con valores entre 7 y 7.1 (Ver figura 5).

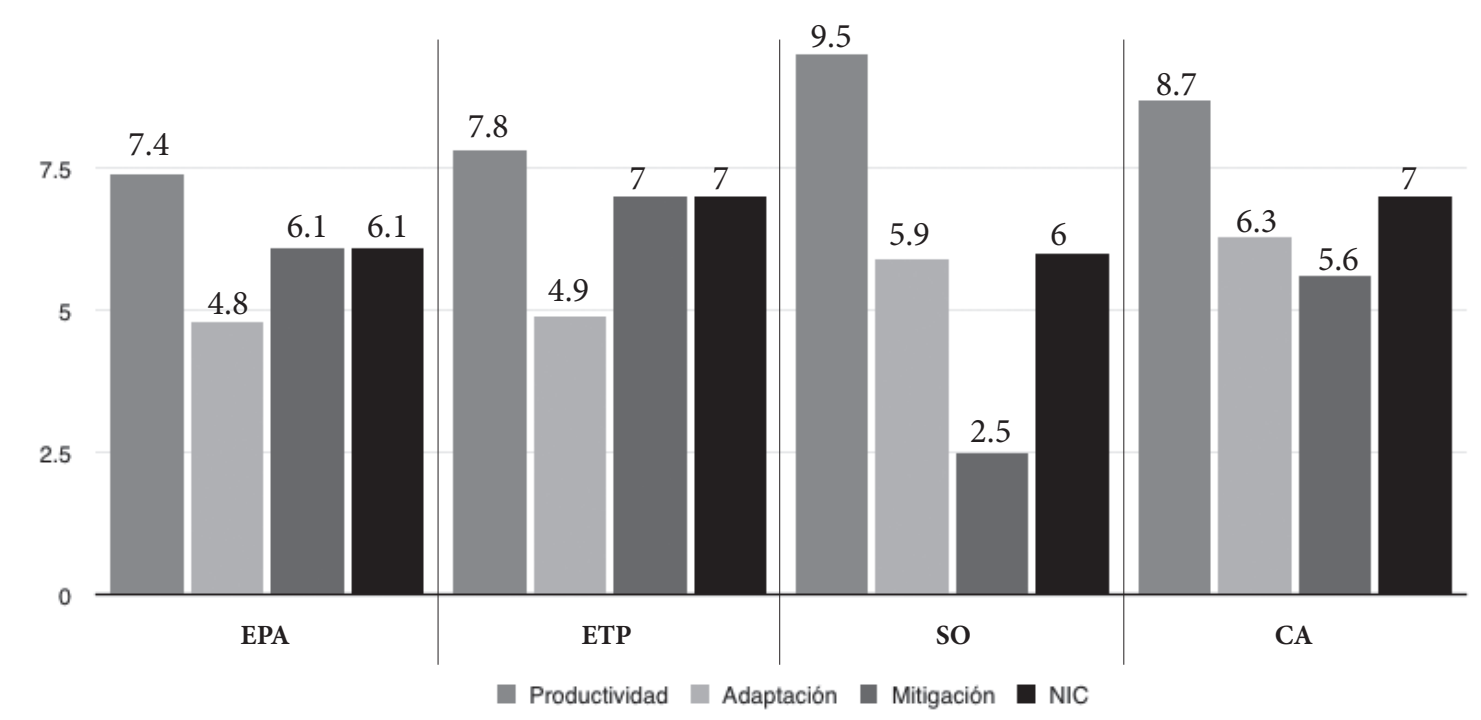

Figura 4. Buenas prácticas en infraestructura identificadas e implementadas de acuerdo con la antigüedad de uso en el municipio de Olanchito, Yoro, Honduras.

SO: salas de ordeño; EPA: espacio para productos agropecuarios; CA: cosechas de agua y ETP: espacio para equipo de trabajo del personal. 


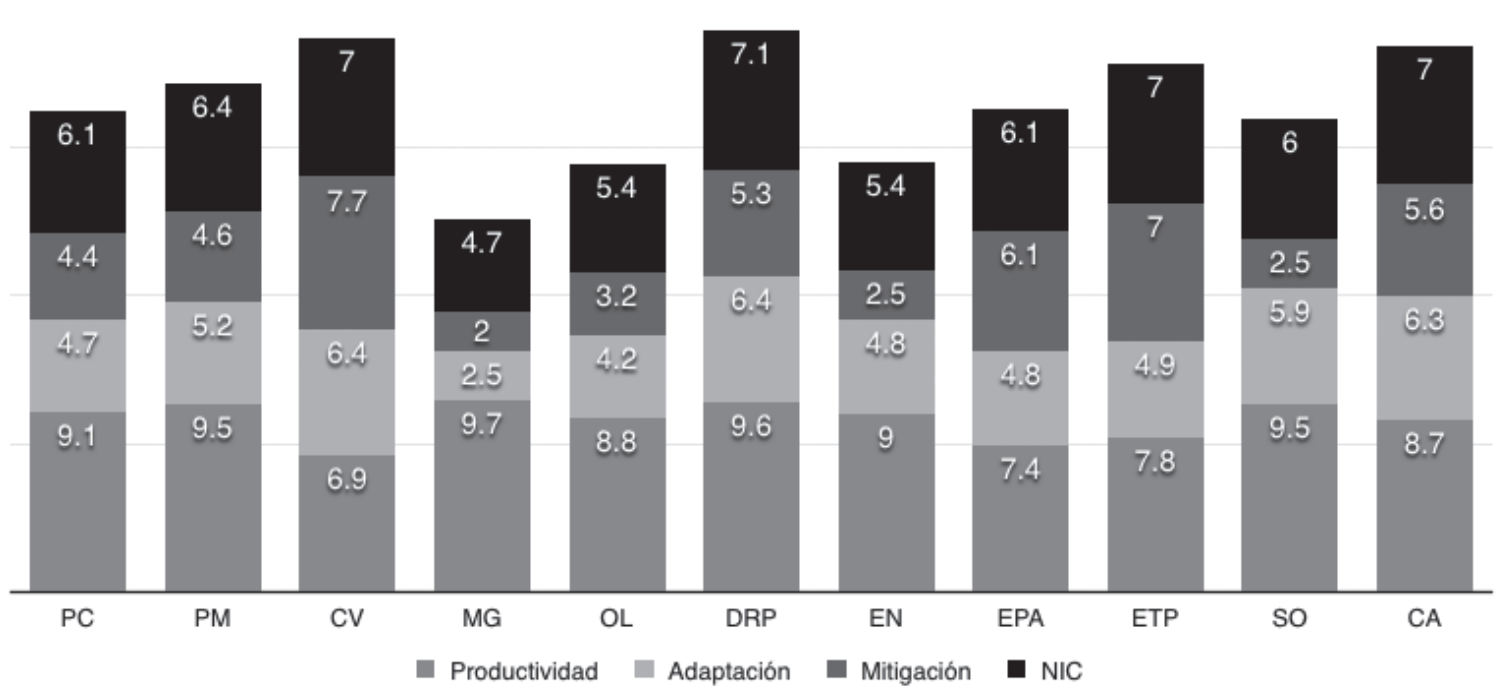

Figura 5. Evaluación cualitativa efectuada a cada una de las prácticas priorizadas a través de los indicadores de productividad, adaptación y mitigación. // PC: pastos de corte; PM: pasturas mejoradas; CV: cercas vivas; MG: mejoramiento genético; DRP: división y rotación de potreros; EN: ensilaje; EPA: espacio para productos agropecuarios; ETP: espacio para equipo de trabajo del personal; SO: salas de ordeño y CA: cosechas de agua.

De acuerdo con el análisis realizado, los productores seleccionan las medidas a implementar considerando en un inicio los costos y beneficios que estas acarrean en sus fincas. Según los escenarios climáticos futuros, esta situación representa una oportunidad para identificar y, posteriormente, promover buenas prácticas de fácil implementación que mejoren las condiciones socioeconómicas de las fincas fomentando la sostenibilidad ambiental, ya que, a diferencia de otros sectores productivos, el sector pecuario emite gas de efecto invernadero, pero cuenta con el potencial para reducir las emisiones (Gerber et al. 2013).

\section{Rentabilidad financiera de fincas que im- plementan buenas prácticas}

En la tabla 2 se presentan los resultados del análisis financiero aplicado en este estudio. Los resultados obtenidos indican que el conjunto de fincas pequeñas tiene los márgenes de ganancias $\left(\$ 977.1 / \mathrm{ha}^{6}\right)$, la tasa de rentabilidad (21.6\%) y el retorno de inversión (B/ $\mathrm{C}=1.5$ ) más elevados que las fincas medianas. Se infiere que estos datos son el resultado de una excelente combinación entre buenas prácticas de SSP, BPM, BPI y de la suplementación. Lo anterior concuerda con lo afirmado por Holguín e Ibrahim (2005), quienes

6. Entiéndase como dólares por hectárea (ha).

Identificación, priorización y análisis costo-beneficio de buenas prácticas ganaderas que los productores de fincas estratificadas implementan para reducir los efectos de la variabilidad climática en el municipio de Olanchito, Yoro, Honduras 
indican que el mejoramiento del sistema de alimentación mediante la inversión en pasturas, bancos forrajeros y suplementación incide positivamente en la obtención de beneficios financieros.

Las fincas medianas tienen gastos mayores en mano de obra y alimentación, lo cual podría deberse a que carecen de conocimiento agropecuario $y$, por ende, las buenas prácticas que implementan no son bien aprovechadas afectando la rentabilidad de las fincas, al igual que lo indicado por Tito (2004) en ganaderías nicaragüenses (Ver tabla 2).

Los resultados de este estudio son superiores y difieren con los reportados por Chavarría et al. (2011), en la subcuenca del río Copán, Honduras; quienes encontraron que las fincas medianas generaron una mayor contribución con un VAN de S384.8/ha y las pequeñas de \$269.7/ha. No obstante, el análisis realizado permite afirmar que, independientemente del número de cabezas y hectáreas disponibles, las actividades de las dos tipologías de fincas son rentables dado que todos los indicadores muestran valores positivos que superan los niveles críticos de decisión.

\section{Análisis financiero por zonas}

El grupo de fincas analizadas en la zona media del valle es el que cuenta con el índice de rentabilidad más alto al obtener ganancias por más de \$900.00/ha. Cuentan con un clima tropical húmedo, la época lluviosa es más extensa, haciendo que las pasturas permanezcan verdes la mayor parte del año; reduciendo el déficit en el alimento base del ganado, representando una situación ventajosa cuando predominan los sistemas extensivos. Este beneficio de las condiciones climáticas hace

Tabla 2. Evaluación financiera por tipología de fincas

\begin{tabular}{|c|c|c|c|c|c|c|c|c|c|c|}
\hline \multirow{2}{*}{$\begin{array}{l}\text { Tipología } \\
\text { de fincas }\end{array}$} & \multirow{2}{*}{$\begin{array}{c}\text { Dólares/ } \\
\text { hectáreas }\end{array}$} & \multicolumn{6}{|c|}{ Años } & \multicolumn{3}{|c|}{$\begin{array}{l}\text { Indicadores } \\
\text { económicos }\end{array}$} \\
\hline & & 1 & 2 & 3 & 4 & 5 & 6 & $\begin{array}{l}\text { VAN/ } \\
\text { ha (\$) }\end{array}$ & TIR\% & BC \\
\hline \multirow{3}{*}{ Pequeñas } & Ingresos & 803.9 & 652.0 & 944.2 & $1,149.5$ & $1,361.3$ & $1,956.1$ & \multirow{3}{*}{977.1} & \multirow{3}{*}{21.58} & \multirow{3}{*}{1.46} \\
\hline & Egresos & $1,934.3$ & 729.6 & 722.9 & 672.3 & 683.8 & 710.0 & & & \\
\hline & Flujo neto & $-1,130.4$ & -77.6 & 221.3 & 477.2 & 677.6 & $1,246.1$ & & & \\
\hline \multirow{3}{*}{ Medianas } & Ingresos & 656.1 & 476.6 & 698.0 & 905.0 & $1,066.9$ & $1,298.9$ & \multirow{3}{*}{728.2} & \multirow{3}{*}{20.71} & \multirow{3}{*}{1.23} \\
\hline & Egresos & $1,779.2$ & 460.5 & 441.5 & 402.8 & 413.7 & 426.6 & & & \\
\hline & Flujo neto & $-1,123.1$ & 16.1 & 256.5 & 502.2 & 653.2 & 872.3 & & & \\
\hline
\end{tabular}

${ }^{\star}$ Nota: VAN/Ha: Valor actual neto por hectárea; TIR: Porcentaje de tasa interna de retorno y B/C: Beneficio/costo. 
que estas fincas inviertan un 20\% menos en la compra de piensos en comparación con las de la zona alta.

Las fincas ubicadas en la zona alta del valle tienen ganancias que sobrepasan los $\$ 700.00$ / ha; sin embargo, el retorno de la inversión es más alto $(\mathrm{B} / \mathrm{C}=1.3)$ que las fincas ubicadas en la zona media (1.2) e igual que las de la zona montañosa (1.3). Los hatos ubicados en la zona alta del valle cuentan con un clima muy seco, con veranos prolongados y pocos meses de lluvia, que pueden ser la causa probable de las ganancias netas obtenidas, ya que es el grupo que invierte más en la compra de insumos externos para la nutrición bovina (un $22 \%$ más que la zona media y $25 \%$ más que las fincas ubicadas en la zona montañosa).
Adicionalmente a la problemática que presenta la zona alta, algunas medidas que ayudarían a contrarrestar los efectos negativos e incrementar las ganancias, como el aumento en la producción láctea y disminución del estrés térmico no son implementadas; un 33\% no utiliza pastos de corte y un $24 \%$ no tiene cercas vivas. En este sentido, las pasturas basadas únicamente en monocultivos y sin cobertura arbórea tienden a degradarse antes de los diez años. Betancourth et al. (2007) señalan que, como consecuencia los ingresos, pueden verse reducidos en un número superior al 50\%, sin considerar los impactos ambientales y sociales relacionados al problema (Ver tabla 3).

Tabla 3. Evaluación financiera por zonas agroclimáticas

\begin{tabular}{|c|c|c|c|c|c|c|c|c|c|c|c|}
\hline \multirow{2}{*}{$\mathbf{n}$} & \multirow{2}{*}{ Zonas } & \multirow{2}{*}{$\begin{array}{l}\text { Dólares/ } \\
\text { hectáreas }\end{array}$} & \multicolumn{6}{|c|}{ Años } & \multicolumn{3}{|c|}{$\begin{array}{l}\text { Indicadores } \\
\text { económicos }^{\star}\end{array}$} \\
\hline & & & 1 & 2 & 3 & 4 & 5 & 6 & $\begin{array}{l}\text { VAN/ } \\
\text { ha (\$) }\end{array}$ & TIR\% & $\mathrm{B} / \mathrm{C}$ \\
\hline \multirow{3}{*}{5} & \multirow{3}{*}{ Alta } & Ingresos & 474.0 & 539.8 & 773.3 & 908.2 & $1,118.6$ & $1,668.7$ & \multirow{3}{*}{776} & \multirow{3}{*}{22.1} & \multirow{3}{*}{1.3} \\
\hline & & Egresos & $1,601.8$ & 503.4 & 533.0 & 490.2 & 489.0 & 517.1 & & & \\
\hline & & Flujo neto & $-1,127.7$ & 36.3 & 240.3 & 417.9 & 629.6 & $1,151.6$ & & & \\
\hline \multirow{3}{*}{6} & \multirow{3}{*}{ Media } & Ingresos & 940.9 & 537.5 & 819.8 & $1,088.6$ & $1,330.5$ & $1,709.5$ & \multirow{3}{*}{917.6} & \multirow{3}{*}{13.5} & \multirow{3}{*}{1.2} \\
\hline & & Egresos & $2,031.3$ & 710.8 & 615.8 & 571.3 & 587.6 & 600.7 & & & \\
\hline & & Flujo neto & $-1,090.4$ & -173.3 & 204.0 & 517.3 & 742.9 & $1,108.8$ & & & \\
\hline \multirow{3}{*}{5} & \multirow[t]{3}{*}{ Montaña } & Ingresos & 586.6 & 556.5 & 757.1 & 938.4 & 958.9 & $1,355.1$ & \multirow{3}{*}{735.5} & \multirow{3}{*}{15.2} & \multirow{3}{*}{1.3} \\
\hline & & Egresos & $1,779.3$ & 349.5 & 433.0 & 391.8 & 409.5 & 301.6 & & & \\
\hline & & Flujo neto & $-1,192.7$ & 207.0 & 324.1 & 546.6 & 549.3 & $1,053.5$ & & & \\
\hline
\end{tabular}

${ }^{\star}$ Nota: VAN/Ha: Valor actual neto por hectárea; TIR\%: Porcentaje de tasa interna de retorno y B/C: Beneficio/costo. 
Es necesario promover el uso de buenas prácticas SSP y ganaderas que han sido evidenciadas en el estudio por los múltiples beneficios económicos y ambientales, que proveen y que ayudan a contrarrestar los efectos adversos del clima (Mahecha 2009). Estudios han comprobado las bondades de los SSP en otros lugares que cuentan con similares condiciones climáticas a la zona alta. Por ejemplo, en el municipio de Apatzingán, México, ubicado a 255msnm y con un trópico seco, se demostraron valores positivos de inversión (VAN, TIR y B/C) en la producción de carne bajo SSP, frente a un sistema tradicional que resultó ser negativo (González Pérez 2015).

Estudios realizados en Costa Rica demuestran que el ganado pastoreado en potreros con alta densidad arbórea (27\%) ganó un peso promedio de $10.4 \mathrm{~kg}$ más que aquellos con baja cobertura (7\%) (Restrepo 2002). De igual manera en Nicaragua, las vacas produjeron en promedio 1L/vaca/día más que aquellas en potreros con baja densidad de árboles (Betancourt et al. 2003). Como se observa, es importante que los productores valoren el efecto de la sombra sobre los rendimientos productivos de la finca.

\section{Análisis financiero por tipología de prácticas}

El grupo de fincas agrupadas en SSP presentan los márgenes de dividendos más altos con ganancias de \$981.00/ha, en comparación con aquellas con buenas prácticas de mane- jo (\$949.3/ha) e infraestructura (\$821.6/ha). Estos valores se aproximan a los encontrados por Chuncho et al. (2012) (VAN \$845.85) en fincas nicaragüenses, que utilizaron tecnologías SSP; y en Petén, Guatemala, donde Turcios et al. (2008) reportaron un VAN \$508.27 (tabla 4).

De acuerdo con Chuncho et al. (2012), esto podría deberse a que la implementación de pastos de corte, pasturas mejoradas y cercas vivas permiten que haya mejores condiciones para la explotación y suplementación de animales. También son un indicador del papel que desempeña el asocio de las tres prácticas. Según Villanueva et al. (2009), los bancos forrajeros contribuyen a la alimentación del animal en épocas críticas donde la disponibilidad y calidad de las pasturas se reduce; los árboles ayudan a reducir el estrés calórico incrementando la producción de leche y carne, y las pasturas mejoradas permiten aumentar la carga animal permitiendo obtener mayores ganancias por hectárea (Holmann et al. 2008).

Estos beneficios fueron identificados por Murgueitio (2009) en algunos países de Latinoamérica. Por ejemplo, este autor observó que al transformar las fincas explotadas con monocultivos a SSP se incrementó el ingreso de los productores desde $\$ 111.00$ a $\$ 180.00$ en Nicaragua; de $\$ 162.00$ a $\$ 252.00$ en Costa Rica, y de $\$ 440.00$ a $\$ 1,597.00$ en Colombia, todos por hectárea por año. (Ver tabla 4). 
Tabla 4. Evaluación financiera por conjunto de buenas prácticas

\begin{tabular}{|c|c|c|c|c|c|c|c|c|c|c|c|}
\hline \multirow{2}{*}{$\mathbf{n}$} & \multirow{2}{*}{$\begin{array}{c}\text { Tipología } \\
\text { de } \\
\text { práctica }^{\star}\end{array}$} & \multirow{2}{*}{$\begin{array}{l}\text { Dólares/ } \\
\text { hectáreas }\end{array}$} & \multicolumn{6}{|c|}{ Años } & \multicolumn{3}{|c|}{$\begin{array}{l}\text { Indicadores } \\
\text { económicos }\end{array}$} \\
\hline & & & 1 & 2 & 3 & 4 & 5 & 6 & $\begin{array}{l}\text { VAN/ } \\
\text { ha (\$) }\end{array}$ & TIR\% & $\mathrm{B} / \mathrm{C}$ \\
\hline \multirow{3}{*}{9} & \multirow{3}{*}{ BPM } & Ingresos & 876.3 & 556.7 & 869.2 & $1,149.5$ & $1,426.5$ & $1,784.7$ & \multirow{3}{*}{949.3} & \multirow{3}{*}{21.0} & \multirow{3}{*}{1.2} \\
\hline & & Egresos & $2,103.0$ & 711.2 & 625.4 & 563.3 & 575.8 & $1,197.4$ & & & \\
\hline & & Flujo neto & $-1,226.7$ & -154.5 & 243.8 & 586.2 & 850.6 & 587.3 & & & \\
\hline \multirow{3}{*}{7} & \multirow{3}{*}{ SSP } & Ingresos & 717.0 & 599.7 & 915.8 & $1,172.7$ & $1,451.8$ & $2,064.8$ & \multirow{3}{*}{980.9} & \multirow{3}{*}{20.1} & \multirow{3}{*}{1.3} \\
\hline & & Egresos & $2,088.5$ & 685.0 & 665.8 & 624.3 & 636.2 & 661.4 & & & \\
\hline & & Flujo neto & $-1,371.5$ & -85.3 & 249.9 & 548.4 & 815.6 & $1,403.4$ & & & \\
\hline \multirow{3}{*}{16} & \multirow{3}{*}{ BPI } & Ingresos & 711.7 & 542.6 & 728.4 & 997.0 & $1,177.7$ & $1,614.6$ & \multirow{3}{*}{821.6} & \multirow{3}{*}{21.8} & \multirow{3}{*}{1.2} \\
\hline & & Egresos & $1,837.6$ & 561.8 & 547.4 & 504.2 & 515.3 & 533.2 & & & \\
\hline & & Flujo neto & -1125.8 & -19.2 & 181.0 & 492.8 & 662.4 & $1,081.3$ & & & \\
\hline
\end{tabular}

Nota: ${ }^{\star S S P}$ : Sistemas silvopastoriles; BPM: Buenas prácticas de manejo y BPI: Buenas prácticas en infraestructura

**VAN/Ha: Valor actual neto por hectárea; TIR\%: Porcentaje de tasa interna de retorno y B/C: Beneficio/costo.

Es importante mencionar que la rentabilidad está asociada con la carga animal. En este sentido, los resultados indicaron que los sistemas de producción que incorporaron SSP, BPM y BPI tienen en promedio 2.3, 1.8 y 1.7UA/ha ${ }^{7}$, respectivamente. En este sentido, otros estudios han demostrado resultados similares; por ejemplo, Chuncho et al. (2012) encontraron que las fincas ganaderas que implementaron SSP tenían un promedio de 1.08UA/ha frente a sistemas convencionales donde fue de $0.82 \mathrm{UA} / \mathrm{ha}$.

Suárez (2009), mediante un estudio realizado en Matagalpa, Nicaragua, demostró un efecto positivo $(\mathrm{p}<0.01)$ entre los bancos forrajeros, pasturas mejoradas $(\mathrm{p}<0.04)$ y la capacidad de carga. La existencia de estos bancos permitió mantener o aumentar la capacidad por unidad de área y/o vaca que le generaron al productor mayores ingresos.

Resaltando las bondades de las prácticas analizadas en este estudio, otros investigadores han demostrado que los SSP ofrecen una serie de beneficios económicos sociales y ambientales. Por ejemplo, además del incremento en la producción de leche (10\% y 20\%) (Ibrahim et al. 2001), con los pastos de corte se promueve la generación de empleo rural al invo-

7. Entiéndase como unidad animal por hectárea (ha).

Identificación, priorización y análisis costo-beneficio de buenas prácticas ganaderas que los productores de fincas estratificadas implementan para reducir los efectos de la variabilidad climática en el municipio de Olanchito, Yoro, Honduras 
lucrar actividades como corte, acarreo, picado y ofrecimiento al ganado (Sánchez 2007); conservación del suelo (Ríos et al. 2007) e incremento de la materia orgánica, fijación de nitrógeno atmosférico, reducción de la emisión de $\mathrm{CH} 4$ y NH4, mejora de las condiciones micro climáticas (Bacab et al. 2013), fijación de carbono (Ibrahim et al. 2007) y biodiversidad (López et al. 2012), entre otros.

\section{Conclusiones}

Los productores han percibido cambios en el clima durante la última década que los relacionan con el aumento o disminución de calor y lluvias; también con los eventos climáticos e impactos generados directamente en la producción ganadera. Los efectos negativos conllevan aumento de enfermedades y plagas en los pastos y animales; disminución de pastoreo, escasez de agua y pastos; bajos rendimientos en la producción, problemas reproductivos y problemas de acceso con respecto a las carreteras, entre otros.

Para enfrentar los eventos climáticos e impactos experimentados en las ganaderías, más de la mitad de los productores han recurrido a la implementación de algunas buenas prácticas silvopastoriles y ganaderas. Por ejemplo, como prácticas SSP, los productores priorizaron el establecimiento de pastos de corte, pasturas mejoradas y las cercas vivas. Como BPM: el mejoramiento genético, ordeño limpio, división y rotación de potreros, y el ensilaje. Como BPI priorizaron un espacio adecuado para guardar productos agropecuarios, así como el equipo del personal de trabajo, la construcción de salas de ordeño y cosecha de agua.

El análisis financiero realizado por tipología de fincas, zonas y tipología de prácticas demostró que todas las inversiones podrían ser rentables, puesto que los indicadores económicos utilizados arrojaron valores positivos. Sin embargo, las fincas analizadas con buenas prácticas SSP generaron mayor ganancia neta $/ \mathrm{ha}^{8}$, mayor rendimiento financiero y una capacidad alta de multiplicar las inversiones realizadas. Esto quiere decir que las ganaderías con buenas prácticas SSP son las que generan mayores beneficios económicos al contribuir con la incorporación de recursos endógenos, que contribuyen a reducir la compra de insumos externos.

8. Entiéndase como ganancia neta por hectárea (ha). 


\section{Referencias}

Acosta, A. y Díaz, T. (ed.). (2014). Lineamientos de política para el desarrollo sostenible del sector ganadero, Organización de las Naciones Unidas para la Alimentación y la Agricultura, Ciudad de Panamá, Panamá, p. 73-80

Alvarado, E. (2005). Modelo de optimización económica para el análisis y simulación de la innovación tecnológica en sistemas de producción de ganado de doble propósito de la región nororiental de Honduras (Tesis de Maestría). Centro Agronómico Tropical de Investigación y Enseñanza, Costa Rica

Andrade, L. (2012). Evaluación de la percepción y los factores determinantes en la implementación de medidas de adaptación al cambio y variabilidad climática por los productores de leche de la cuenca del río La Villa, Panamá (Tesis de Maestría). Centro Agronómico Tropical de Investigación y Enseñanza, Costa Rica

Bacab, M.; Madera, B.; Solorio, J.; Vera, F. y Marrufo, F. (2013). Los sistemas silvopastoriles intensivos con Leucaena leucocephala: una opción para la ganadería tropical. Avances en Investigación Agropecuaria, 17(3), 67-81

Balana, B.; Muys, M.; Haregeweyn, N.; Descheemaeker, K.; Deckers, J.; Poesen, J.; Nyssen, J. y Mathijs E. (2012). Cost-benefit analysis of soil and water conservation measure: the case of exclosures in northern Ethiopia. For. Pol. Econ. 15, 27-36

Banegas, K.; Moscoso, C.; Ibrahim, M.; Nieuwenhyse, A. y Gutiérrez, I. (2013). Principales cambios en las fincas ganaderas de productores que participaron en escuelas de campo de la región Trifinio, VII Congresso Latinoamericano de Sistemas Agroflorestais para a Producao Pecuária Sustentável, Centroamérica, 822-827

Barrios, C. (2008). Estudio de mercado de productos pecuarios en tres zonas piloto del Proyecto Desarrollo Participativo de Alternativas de Uso sostenible de la tierra Para Áreas de Pasturas degradadas en América Central (CATIE/Noruega-Pasturas Degradadas). Centro Agronómico Tropical de Investigación y Enseñanza, Turrialba, Costa Rica. Recuperado de http://repositorio.bibliotecaorton.catie.ac.cr/bitstream/handle/11554/7954/528. pdf? sequence $=2$ \&isAllowed $=y$

Identificación, priorización y análisis costo-beneficio de buenas prácticas ganaderas que los productores de fincas estratificadas implementan para reducir los efectos de la variabilidad climática en el municipio de Olanchito, Yoro, Honduras 
Betancourt, K.; Ibrahim, M.; Harvey, C. y Vargas, B. (2003). Efecto de la cobertura arbórea, sobre el comportamiento animal en fincas ganaderas de doble propósito en Matiguás, Matagalpa, Nicaragua. Agroforestería en las Américas 10(39-40), 47-51

Betancourth, H.; Pezo, D.; Cruz, J. y Beer, J. (2007). Impacto bioeconómico de la degradación de pasturas en fincas de doble propósito en el Chal, Petén, Guatemala. Pastos y forrajes 30(1), 169-177.

Birol, E.; Koundouri, P. y Kountouris Y. (2010). Assessing the economic viability of alternative water resources in water-scarce regions: combining economic valuation, cost-benefit analysis and discounting. Ecol. Econ. 69(4), 839-847.

Bizoza, R. y De Graaff, J. (2012). Financial cost-benefit analysis of bench terraces in Rwanda. Land Degrad. Dev. 23(2), 3-115.

Chuncho, C.; Sepúlveda, C.; Ibrahim, M.; Chacón, A.; Benjamín, T. y Tobar D. (2012). Percepción y medidas de adaptación al cambio climático implementadas en época seca para ganaderos en río Blanco y Paiwas, Nicaragua. Revista CEDAMAZ 2(1), 78-91

Climate Change, Agriculture and Food Security (2015). Marco para la priorización de inversiones en agricultura sostenible adaptada al clima. Recuperado de https://ccafs.cgiar.org/ es/publications/marco-para-la-priorizaci\%C3\%B3n-de-inversiones-en-agricultura-sostenible-adaptada-al-clima-0\#.Wg49jkribIV

Collier, R.J. y Zimbelman, R.B. (febrero 22-23, 2007). Heat stress effects on cattle: what we know and what we don't know. 22nd Annual Southwest Nutrition \& Management Conference. Department of Animal Sciences The University of Arizona, Tucson, Arizona, 76-83 
Costa Rica, Centro Agronómico Tropical de Investigación y Enseñanza - Aguilar, A.; Cruz, J.; Flores, J.; Nieuwenhuyse, A.; Pezo, D. y Piniero, M. (2010). ¿Cómo trabajar con las familias ganaderas y las organizaciones de investigación y desarrollo para lograr una ganadería más sostenible y productiva? Las experiencias del proyecto CATIE-Noruega/Pasturas Degradadas con procesos de aprendizaje participativo en Centroamérica. División de Investigación y Desarrollo, Turrialba. Recuperado de http://orton.catie.ac.cr/REPDOC/ A5978E/A5978E.PDF

Craine, M.; Elmore, O. y Tolleson, D. (2010). Climate change and cattle nutritional stress. Global Change Biology 16, 2901-2911.

Daigneault, A.; Brown, P. y Gawith, D. (2016). Dredging versus hedging: Comparing hard infrastructure to ecosystem-based adaptation to flooding. Ecol. Econ. 122, 25-35. Recuperado de http://dx.doi.org/10.1016/j.ecolecon.2015.11.019

Dittrich, R.; Wreford, A. y Moran, D. (2016). A survey of decision-making approaches for climate change adaptation: are robust methods the way forward. Ecol. Econ. 122, 79-89. Recuperado de http://dx.doi.org/10.1016/j.ecolecon.2015.12.006

Escobar, P.; Mader, T. y Arias, R. (2008). Climatic factors affecting cattle performance in dairy and beef farms (en línea). Arch Med Vet 40 (7-22)

Estados Unidos, Department of Agricultute - Rose, D.; Blinn, C. y Brand, G. (1988). A guide to forestry investment analysis.

Food and Agriculture Organization of the United Nations (2014). Anuario Estadístico de la FAO: La Alimentación y la Agricultura en América Latina y el Caribe; Roma, Italia

Gerber, P.; Steinfeld, H.; Henderson, B.; Mottet, A.; Opio, C.; Dijkman, J.; Falcucci, A. y Tempio, G. (2013). Tackling climate change through livestock: a global assessment of emissions and mitigation opportunities. FAO. Roma, Italia

González, J.M. (2015). Evaluación económica de una engorda de toretes en dos sistemas de alimentación. CIENCIA 23(2), 154-162 
Hatfield, J.L.; Boote, K.J.; Kimball, B.A.; Ziska, L.H.; Izaurralde, R.C.; Thomson, A.M. y Wolfe, D. (2011). Climate impacts on agriculture: implications for crop production. Agronomy Journal 103(2), 351-370

Hartter, J.; Stampone, M.D.; Ryan, S.J; Kirner, K.; Chapman, C.A. y Goldman, A. (2012). Patterns and Perceptions of Climate Change in a Biodiversity Conservation. Hotspot. PloS one $7(2)$

Hernández, G.; Villanueva, C.; Medina, J.; Tobar, D. y Louman, B. (2014). Buenas prácticas para la adaptación al cambio climático en fincas ganaderas de Honduras. Centro Agronómico Tropical de Investigación y Enseñanza; Managua, Nicaragua

Holguín, V. y Ibrahim, M. (2005). Bancos forrajeros de especies leñosas. Centro Agronómico Tropical de Investigación y Enseñanza; Costa Rica

Honduras, Secretaría de Recursos Naturales y Ambiente (2012). Segunda Comunicación Nacional del Gobierno de Honduras ante la Convención Marco de las Naciones Unidas sobre Cambio Climático. Tegucigalpa

Horngren, Ch.; Datar, S. y Foster, G. (2007). Contabilidad de Costos. Un enfoque gerencial. Decimosegunda edición: México, Pearson Prentice Hall

Ibrahim, M.; Chacón, M.; Cuartas, C.; Naranjo, J.; Ponce, G.; Vega, P.; Casasola, F. y Rojas, J. (2007). Almacenamiento de carbono en el suelo y la biomasa aérea en sistemas de uso de la tierra en paisajes ganaderos de Colombia, Costa Rica y Nicaragua. Agroforestería en las Américas 45, 27-36 
Ibrahim, M.; Franco, M.; Pezo, D.; Camero, A. y Araya, J. (2001). Promoting intake of Cratylia Argentea as a dry season suplement for cattle grazing hyparrhenia ruffa in the subhumids tropics of Costa Rica. Agroforestry systems (51), 167-175

Instituto de Conservación y Desarrollo Forestal, Áreas protegidas y vida silvestre - The Nature Conservancy (2009). Plan de manejo del área hábitat/Especie del Colibrí Esmeralda Hondureño Amazilia Luciae. Recuperado de http://icf.gob.hn/wp-content/uploads/2015/09/ AP-Plan-de-Manejo-CEH.pdf

Instituto Nacional de Estadística (2013). Censo de XVII población y vivienda. Información del municipio. Recuperado de http://www.redatam.org/binhnd/RpWebEngine.exe/ Portal?BASE=MUNDEP18\&lang=ESP

Izaurralde, R.C.; Thomson, A.M.; Morgan, J.A.; Fay, P.A.; Polley, H.W. y Hatfield, J.L. (2011). Climate impacts on agriculture: implications for forage and rangeland production. Agronomy Journal 103(2), 371-381

López, E.; Solorío, F.; Chay, A.; González, A.; Ku-Vera, J. y Ramírez, L. (2012). Indicadores ambientales y biodiversidad de fauna en sistemas silvopastoriles intensivos en el Valle de Tepalcatepec, Michoacán, IV Congreso Internacional sobre Sistemas Silvopastoriles Intensivos. Fundación Produce Michoacán, Morelia, México

Mahecha, L. (2009). El silvopastoreo: una alternativa de producción que disminuye el impacto ambiental de la ganadería bovina. Revista Colombiana de Ciencias Pecuarias 15(2), 226-231

Mosquera, D. (2010). Conocimiento local sobre bienes y servicios de especies arbóreas y arbustivas en sistemas de producción ganadera de Rivas, Nicaragua. (Tesis de Maestría). Centro Agronómico Tropical de Investigación y Enseñanza, Turrialba, Costa Rica

Murgueitio, E. (2009). Incentivos para los sistemas silvopastoriles en América Latina. Avances en Investigación Agropecuaria 13(1), 3-19

Nardone, A.; Ronchi, B.; Lacetera, N.; Ranieri, S. y Bernabucci, U. (2010). Effects of climate changes on animal production and sustainability of livestock systems. Livestock Science $130,57-69$ 
Pezo, D. (2009). Los pastizales seminaturales de América Central. Un recurso forrajero poco estudiado. Agroforestería en las Américas (47), 4-5

Prabuddh, K. y Suresh, C. (2014). A cost-benefit analysis of indigenous soil and water conservation measures in Sikkim Himalaya India. Mt. Res. Dev. 34(1), 27-35. Recuperado de http://dx.doi.org/10.1659/MRD-JOURNAL-D-12-00013.1

Restrepo, C. (2002). Relaciones entre la cobertura arbórea en potreros y la producción bovina en fincas ganaderas en el trópico seco, Cañas, Costa Rica (Tesis de maestría), Centro Agronómico Tropical de Investigación y Enseñanza, Turrialba, Costa Rica

Riera, C. y Pereira S. (2013). Between climate risk and productive changes: irrigated agriculture as a form of adaptation in Río Segundo, Córdoba, Argentina. Investigaciones Geográficas: Boletín - Instituto de Geografía, Universidad nacional Autónoma de México (82), 52-65. Recuperado de https://doi.org/10.14350/rig.33718

Ríos, N.; Cárdenas, A.; Andradre, H.; Ibrahim, M.; Jiménez, F.; Sancho, F.; Ramírez, E.; Reyes, B. y Woo, A. (2007). Estimación de la escorrentía superficial e infiltración en sistemas de ganadería convencional y en sistemas silvopastoriles en el trópico subhúmedo de Nicaragua y Costa Rica. Agroforestería en las Américas 45, 66-71

Rust, M. y Rust, T. (2013). Climate change and livestock production: A review with emphasis on Africa. South African Journal of Animal Science. 43(3), 56-267

Sáenz, J.C.; Villatoro, F.; Ibrahim, M.; Fajardo, D. y Pérez, M. (2007). Relación entre las comunidades de aves y la vegetación en agro paisajes dominados por la ganadería en Costa Rica, Nicaragua y Colombia. Agroforestería en las Américas 45, 37-48

Sánchez, L. (2007). Caracterización de la mano de obra en fincas ganaderas y rentabilidad de bancos forrajeros en Esparza, Costa Rica. (Tesis de maestría). Centro Agronómico Tropical de Investigación y Enseñanza, Turrialba, Costa Rica

Sánchez, B. (2014). Sistemas silvopastoriles en Honduras: Una Alternativa para mejorar la ganadería, Organización de las Naciones Unidas para la Alimentación y la Agricultura, Tegucigalpa, Honduras 
Seo, S.N.; McCarl, B.A. y Mendelsohn, R. (2010). From beef cattle to sheep under global warming? An analysis of adaptation by livestock species choice in South America. Ecological Economics 69(12), 2486-2494.

Sepúlveda, C.; Marín, Y. y Ibrahim, M. (2008). Adaptación al cambio climático y percepción de ganaderos en Costa Rica y Nicaragua

Suárez, J. (2009). Análisis de rentabilidad en los sistemas tradicionales de producción y la incorporación de los sistemas silvopastoriles en fincas de doble propósito Matagalpa-Nicaragua. (Tesis de maestría), Centro Agronómico Tropical de Investigación y Enseñanza, Turrialba, Costa Rica

Swisscontact (2014). Análisis de la cadena de valor bovino láctea en el golfo de fonseca, Honduras. Tegucigalpa, Honduras

Tito, M. (2004). Efectos de la incorporación de tecnologías silvopastoriles sobre la demanda de mano de obra y la rentabilidad de las fincas ganaderas de Muy, Nicaragua (Tesis de maestría), Centro Agronómico Tropical de Investigación y Enseñanza, Turrialba, Costa Rica

Turcios, S.H. (2008). Evaluación del proceso de toma de decisiones para adopción de bancos de proteína de leucaena (Leucaena leucocephala) y su efecto como suplemento nutricional para vacas lactantes en sistemas doble propósito en El Chal, Petén, Guatemala, (Tesis de maestría), Centro Agronómico Tropical de Investigación y Enseñanza, Turrialba, Costa Rica

United Nations Environment Programme (2016). The Adaptation Finance Gap Report. Recuperado de http://web.unep.org/adaptationgapreport/sites/unep.org.adaptationgapreport/ files/documents/agr2016.pdf

Villanueva, C.; Ibrahim, M. y Casasola, F. (2008a). Valor económico y ecológico de las cercas vivas en fincas y paisajes ganaderos, Centro Agronómico Tropical de Investigación y Enseñanza, Turrialba, Costa Rica

Villanueva, C.; Ibrahim, M.; Torres, K. y Torres, M. (2008b). Planificación agroecológica de fincas ganaderas: La experiencia de la subcuenca Copán, Honduras. Centro Agronómico Tropical de Investigación y Enseñanza, Turrialba, Costa Rica. Recuperado de http://www. sidalc.net/repdoc/A2984E/A2984E.PDF

Identificación, priorización y análisis costo-beneficio de buenas prácticas ganaderas que los productores de fincas estratificadas implementan para reducir los efectos de la variabilidad climática en el municipio de Olanchito, Yoro, Honduras 
Villanueva, C.; Ibrahim, M.; Casasola, F.; Ríos, N. y Sepúlveda, C. (2009c). Políticas y sistemas de incentivo para el fomento y adopción de buenas prácticas agrícolas como una medida de adaptación al cambio climático en América Central, 103 - 125

Villanueva, C.; Ibrahim, M. y Haensel, G. (2010d). Producción y rentabilidad de sistemas silvopastoriles. Estudios de caso en América Central. Centro Agronómico Tropical de Investigación y Enseñanza, Turrialba, Costa Rica 